\title{
ORTHOGONAL GROUP IN A GALOIS FIELD.
}

BY DR. L. E. DICKSON.

(Read before the American Mathematieal Society at the Meeting of De cember 29, 1897.)

1. A linear substitution $S$ on the marks of a Galois Field of order $p^{n}$ (in notation $G F\left[p^{n}\right]$ )

$$
\xi_{i}^{\prime}=\sum_{j=1}^{m} \alpha_{i j} \xi_{j} \quad(i=1,2, \cdots m)
$$

will be called orthogonal if it leaves absolutely invariant

$$
\xi_{1}^{2}+\xi_{2}^{2}+\cdots+\xi_{m}^{2} .
$$

The conditions on the coefficients of $S$ are seen to be

$$
\begin{array}{r}
\alpha_{1 j}{ }^{2}+\alpha_{2 j}{ }^{2}+\cdots+\alpha_{m j}{ }^{2}=1 \quad(j=1, \cdots m), \\
\alpha_{1 j} \alpha_{1 k}+\alpha_{2 j} \alpha_{2 k}+\cdots+\alpha_{m j} \alpha_{m k}=0 \quad(j, k=1, \cdots m, j \neq k),
\end{array}
$$

the latter not occurring* if $p=2$. Replacing $\alpha_{i j}$ by $\alpha_{i i}$ we get the reciprocal of $S$, with a set of conditions equivalent to the above. Thus the determinant of $S^{-1}$ equals the determinant $A$ of $S$, so that $A^{2}=1$, being the determinant of $S^{-1} S$.

2. For the case $p=2$, an orthogonal substitution $S$ leaves invariant the square root of $\xi_{1}{ }^{2}+\cdots+\xi_{m}{ }^{2}$ in the $G F\left[2^{n}\right]$, viz.,

$$
X \equiv \xi_{1}+\xi_{2}+\cdots+\xi_{m} .
$$

Taking as independent indices $X, \xi_{2}, \cdots \xi_{m}, S$ takes the form (with unaltered determinant $A=1$ ):

$$
X^{\prime}=X, \quad \xi_{i}^{\prime}=\sum_{j=2}^{m} \beta_{i j} \xi_{j}+\alpha_{i 1} X \quad(i=2, \cdots m),
$$

where the $\alpha_{i 1}$ are arbitrary and the $\beta_{i j} \equiv \alpha_{i j}+\alpha_{i 1}$ satisfy the condition

$$
A=\left|\beta_{i j}\right|=1 \quad(i, j=2, \cdots m) .
$$

The order of the orthogonal group $G$ on $m$ indices in the $G F\left[2^{n}\right]$ is thus

$$
2^{n(m-1)}\left(\frac{\left(2^{n(m-1)}-1\right)\left(2^{n(m-1)}-2^{n}\right) \cdots\left(2^{n(m-1)}-2^{n(m-2)}\right)}{2^{n}-1}\right),
$$

* The remark of Jordan, Traité des Substitutions, p. 169, 11. 18-21, is thus not exact. 
the quantity in brackets being the order of the group $* I$ of substitutions of determinant 1 on $m-1$ indices of the $G F\left[2^{n}\right] . \quad G$ is obtained by extending $\Gamma$ by the substitutions

$$
\xi_{i}^{\prime}=\xi_{i}+\gamma_{i} X, X^{\prime}=X,
$$

forming a commutative group self-conjugate under $G$. Hence the decomposition of $G$ reduces to that of $\Gamma$ (reference just given). Henceforth I suppose $+p \neq 2$.

3 . We may readily generalize Jordan, $\$ \S 197-199$, thus:

TheOREM: The number of systems of solutions in the $G F\left[p^{n}\right]$, $p \neq 2$, of

$$
\alpha_{1} \xi_{1}^{2}+\alpha_{2} \xi_{2}^{2}+\cdots+\alpha_{2 m} \xi_{2 m}^{2}=x,
$$

where every $\alpha_{j}$ is a mark $\neq 0$ of the $G F\left[p^{n}\right]$, is

$$
\begin{array}{ll}
p^{n(2 m-1)}-p^{n(m-1) \nu} & (\% \neq 0) \\
p^{n(2 m-1)}+\left(p^{n m}-p^{n(m-1)}\right) \vee & (\%=0),
\end{array}
$$

where $\nu$ is +1 or -1 according as $(-1)^{m} \alpha_{1} \alpha_{2} \cdots \alpha_{2 m}$ is a square or not square in the $G F\left[p^{n}\right]$.

Similarly from $\$ 200$ (where the minus sign is a misprint):

THEOREM : The number of systems of solutions of

$$
\alpha_{1} \xi_{1}^{2}+\alpha_{2} \xi_{2}^{2}+\cdots+\alpha_{2 m+1} \xi_{2 m+1}^{2}=\varkappa
$$

is $p^{2 n m}+p^{n m} v^{\prime}$, where $v^{\prime}$ is $+1,-1$, or 0 according as $(-1)^{m} a_{1} a_{2} \cdots \alpha_{2 m+1} *$ is a square, not-square or zero in the $G F\left[p^{n}\right]$.

4. In view of the succeeding paragraphs, it may be readily seen that the investigation of Jordan, $\$ \$ 201-212$, affords the following generalization :

The orthogonal group on $m$ indices in the $G F\left[p^{n}\right], p \neq 2$ is generated \$ by the substitutions [only the indices changed being written]:

$$
\xi_{i}^{\prime}=\alpha \xi_{i}+\beta \xi_{j}, \quad \xi_{j}^{\prime}=-\beta \xi_{i}+\alpha \xi_{j} \quad\left(\alpha^{2}+\beta^{2}=1\right)
$$

and

$$
\xi_{i}^{\prime}=-\xi_{i}
$$

*Current number of the Annals of Mathematics, article on linear groups. $\dagger$ Note the correction of Jordan, p. 169, 1. 15, in either of the ways:

$$
\begin{aligned}
& |x, y, z . u, v \quad y+z+u, x+z+u, z, u, v| \\
& |x, y, z, u, v \quad y+z+u, x+u+v, x+y+u, y, x| .
\end{aligned}
$$

$\ddagger$ The only exception is $p^{n}=5$, when other generators are necessary if $m>2$. Thus, for $m=3$, we may choose the additional generator

$$
\xi_{1}^{\prime}=2 \xi_{1}+\xi_{2}+\xi_{3}, \quad \xi_{2}^{\prime}=\xi_{1}+2 \xi_{2}+\xi_{3}, \quad \xi_{3}^{\prime}=\xi_{1}+\xi_{2}+2 \xi_{3} .
$$


and its order is $P_{m} \cdot P_{m-1} \cdots P_{1}$, where $P_{t}$ denotes the number of solutions in the $G F^{m}\left[p^{n}\right]$ of $\xi_{1}{ }^{2}+\xi_{2}{ }^{2}+\cdots+\xi_{t}^{2}=1$, given by $\$ 3$.

Hence if $\varepsilon=+1$ or -1 according as $-1=$ square or not-square, we have

$$
\begin{gathered}
P_{4 t}=p^{n(4 t-1)}-p^{n(2 t-1)} ; P_{4 t+1}=p^{4 n t}+p^{2 n t} ; \\
P_{4 t+2}=p^{n(4 t+1)}-\varepsilon p^{2 n t} ; P_{4 t+3}=p^{2 n(2 t+1)}+\varepsilon p^{n(2 t+1)} . \\
\text { Thus } \quad P_{4 t+2} \cdot P_{4 t+3}=p^{n(4 t+1)}\left(p^{n(4 t+2)}-1\right) .
\end{gathered}
$$

Except when $m=4 t+2$, the order of the orthogonal group on $m$ indices is independent of the quadratic character of -1 .

If $m=2 k+1$ the order is $2 \omega$, where $\omega$ is the order of the linear Abelian group on $2 k$ indices (with the factors of composition 2 and $\omega / 2$ ), viz.:

$$
\omega=\left(p^{2 n k}-1\right) p^{n(2 k-1)}\left(p^{n(2 k-2)}-1\right) p^{n(2 k-3)} \cdots\left(p^{2 n}-1\right) p^{n} .
$$

5. To generalize Jordan, $\$ \$ 208-9$, we need the theorem: In every GF $\left[p^{n}\right]$, except for $p^{n}=3^{2}, 5$ or 13 , a mark $\nu$ may be found, such that $\nu^{4}-1$ shall be at wish a square or a not-square.

For $n=1$ this theorem was proved by Gauss.* Thus, if $p \neq 5$ or 13 (exceptions omitted by Jordan), an integer $\nu \neq 0$ exists, making $\nu^{4}-1$ a square in the $G F\left[p^{1}\right]$ and hence also a square in the $G F\left[p^{n}\right]$; likewise an integer $\nu^{4}-1$ exists which is a not-square in the $G F\left[p^{1}\right]$ and hence in the $G F\left[p^{n}\right], n$ odd. For the case $n$ even, and thus $p^{n}=$ $8 t+1$, we may readily generalize Gauss, 1. c. 16-18, and obtain the formulæ:

$$
\begin{gathered}
2 k=i+l, m=-k+\left(p^{n}-1\right) / 8, p^{n}=[4(k-m)+1]^{2}+ \\
4(l-i)^{2},
\end{gathered}
$$

from which we are to prove that (in Gauss' notation) $i \equiv(10)$ and $l \equiv(30)$ are not both zero. But if $i=l=0$, we readily find

$$
\left( \pm p^{\frac{n}{2}}-1\right)^{2}=4 \quad \text { or } p^{n}=3^{2} .
$$

The proposition fails for the $G F\left[3^{2}\right]$, which we may define by the irreducible congruence $j^{2} \equiv-1(\bmod 3)$. Thus $j+1$ is a primitive root and Gauss' four classes are

$$
1,-1 ; j+1,-j-1 ;-j, j ;-j+1, j-1 \text {; }
$$

* Theoria residuorum biquadraticorum commentatio prima, 16-21.

+ If $p$ be of the form $4 t+1$, so that $p^{n}$ may be expressed as the sum of two squares each $\neq 0$, the proof follows as in Gauss, Art. 18 , since $l \neq i$. 
the fourth powers are $1,-1$ and thus neither is followed (on adding +1 ) by a not-square. But for $p^{n}=3^{2}$, the theorem of Jordan, \$208, follows by $\$ 203$ since

$$
1-c^{\prime \prime 2}=\alpha^{\prime 2}+b^{\prime 2}=1+1=-1=\text { square. }
$$

It remains to prove the theorem for $5^{n \prime}$ and $13^{n \prime}, n^{\prime}$ odd and $>1$. Consider the general case $p^{n \prime}=8 n+5$. By Gauss, Art. 20 generalized, there exist $2 h$ squares and $2 m$ not-squares each followed by a fourth power. But $h=0$ gives $m=n, i+l=1, k=2 n$, whence

$$
p^{n \prime}=8 n+5=(-4 n+1)^{2}+4 \text {. }
$$

Hence $n=0$ or 1 , so that $p^{n \prime}=5$ or 13 . Again, $m=0$ gives $h+k=0, h=n$, so that $p^{n \prime}=5$. That $p^{n \prime}=5$ and 13 are really exceptions appears at once from the tables of Gauss, Art. 15.

For $p=13$ the result of Jordan $\$ 208$ may be obtained as follows. We have $\alpha^{\prime}= \pm 1, b^{\prime}= \pm 1, c^{\prime \prime}= \pm 5$. Similarly, as in $\$ 204$, I take $\beta b^{\prime}-\gamma c^{\prime \prime}=b^{\prime \prime}$. Then for $\beta= \pm 2$, $-\gamma= \pm 6$, the signs to agree with those of $b^{\prime}$ and $c^{\prime \prime}$ respectively, we have $b^{\prime \prime}=2+30,1-b^{\prime \prime 2}=4$, a case solved by $\$ 203$.

The proof needed in $\$ 209$ follows as a corollary if $p^{n}+3^{2}$ or 5. Thus if $\nu^{4}-1$ and hence also $1-\nu^{4}$ be a not-square, either at once $1-\nu^{2}$ is a not-square and $1+\nu^{2}$ a square, or vice versa, when we replace $\nu$ by $\nu \sqrt{-1},-1$ being a square. But if $p^{n}=3^{2}$, we cannot proceed as in $\$ 209$. Since $a^{\prime}= \pm 1, b^{\prime}= \pm d, 1-d^{2}=$ not-square, we must have

Thus

$$
\begin{aligned}
d^{2}= \pm j, & c^{\prime \prime 2}=\mp j \\
b^{\prime}= \pm(j-1), & c^{\prime \prime}= \pm(j+1)
\end{aligned}
$$

or vice versa, leading to a similar treatment. As in $\$ 204$, I take

$b^{\prime \prime}=\beta b^{\prime}-\gamma c^{\prime \prime}=\beta[ \pm(j-1)]-\gamma[ \pm(j+1)], \quad\left(\beta^{2}+\gamma^{2}=1\right)$.

We may take $\beta= \pm j, \gamma= \pm j$ such that the signs combine to give

$$
b^{\prime \prime}=j(j-1)-j(j+1)=-2 j,
$$

whence $1-b^{\prime 2}=-1=$ square, a case solved by $\$ 203$.

6. For $\$ \$ 207$ we need the theorem, proved as in Jordan, $\S 198$ or as in Gauss, 1. c. Art. 16:

In the $G F\left[p^{n}\right]$, for which $-1=$ square, $\left(p^{n}-5\right) / 4$ of the squares are followed by squares, $\left(p^{n}-1\right) / 4$ by not-squares, and one (viz., -1) by zero. 
7. As in $\$ 210, p^{2 n}+4 p^{n}-1$, being relatively prime to $p$, must divide $\left(p^{3 n}-1\right)\left(p^{2 n}-1\right)$ and thus also $4 p^{n}\left(p^{3 n}-1\right)$ and hence* $4\left(17 p^{n}-5\right)$ and hence divides

$$
20\left(p^{2 n}+4 p^{n}-1\right)-\left(68 p^{n}-20\right)=p^{n}\left(20 p^{n}+12\right)
$$

Hence $\left(p^{n}+2\right)^{2}-5$ must divide 304 , since

Thus

$$
\begin{gathered}
3\left(68 p^{n}-20\right)+5\left(20 p^{n}+12\right)=304 p^{n} . \\
p^{n}+2<18>\sqrt{309 .}
\end{gathered}
$$

But $p^{n}=13,11,9,5,3$ are readily excluded; while $p^{n}=7$ yields 76 a divisor of 304 and indeed of $\left(7^{3}-1\right)\left(7^{2}-1\right)$, but is excluded since -1 is a non-residue of 7 .

8. With the hypothesis of Jordan $\$ 211$, that $a^{2}+b^{2}+c^{2}=0$, etc., we have $a^{2}=b^{2}=\cdots$. Hence $3 a^{2}=3 b^{2}=\cdots=0$ and $m a^{2}=1$. Thus either $a^{2}=b^{2}=\cdots=1$ or $2 a^{2}=2 b^{2}=\cdots=1$, when $1-a^{2}=a^{2}=$ square.

UNIVERSITY OF CALIFORNIA, November $20,189 \%$.

\section{WEBER'S ALGEBRA.}

Lehrbuch der Algebra. By Heinrich WeBer, Professor of Mathematics in the University of Strassburg. Braunschweig, Friedrich Vieweg und Sohn. 1895-96. 8vo. Vol. I., pp. 653 ; Vol. II., pp. 796.

For some years the need of a thoroughly modern textbook on algebra has been seriously felt. The great strides that algebra has taken during the last twenty-five years, in almost all directions, have made Serret's classical work more and more antiquated, while modern books like Petersen's and Carnoy's make no claims to give a large and comprehensive survey of the subject. The appearance of any book modelled on the same broad plan as Serret's Algèbre Supérieure would thus be greeted with a hearty welcome, but when written by such a master as Heinrich Weber, we greet it with expressions of sincerest joy and satisfaction.

As Weber himself tells us, he has cherished for years the plan of this great undertaking; but before deciding to execute it he has traversed in his university lectures many times this vast domain as a whole, and has treated various parts separately with greater detail. No wonder, then, that

\footnotetext{
* Jordan has $68 p-12$, thus losing the divisor 76 .
} 